\title{
Article \\ Study of 3D-Printed Dielectric Barrier Windows for Microwave Applications
}

\author{
Mikhail D. Proyavin D, Dmitry I. Sobolev, Vladimir V. Parshin, Vladimir I. Belousov, Sergey V. Mishakin \\ and Mikhail Y. Glyavin *D
}

Institute of Applied Physics of the Russian Academy of Sciences, 603950 Nizhny Novgorod, Russia; pmd@ipfran.ru (M.D.P.); sobolev@ipfran.ru (D.I.S.); parsh@ipfran.ru (V.V.P.); vbelousov@ipfran.ru (V.I.B.); mishakin@ipfran.ru (S.V.M.)

* Correspondence: glyavin@ipfran.ru

Citation: Proyavin, M.D.; Sobolev, D.I.; Parshin, V.V.; Belousov, V.I.; Mishakin, S.V.; Glyavin, M.Y. Study of 3D-Printed Dielectric Barrier Windows for Microwave Applications. Electronics 2021, 10, 2225. https://doi.org/10.3390/ electronics10182225

Academic Editor: Anna B. Piotrowska

Received: 30 June 2021

Accepted: 9 September 2021

Published: 10 September 2021

Publisher's Note: MDPI stays neutral with regard to jurisdictional claims in published maps and institutional affiliations.

Copyright: (C) 2021 by the authors. Licensee MDPI, Basel, Switzerland. This article is an open access article distributed under the terms and conditions of the Creative Commons Attribution (CC BY) license (https:/ / creativecommons.org/licenses/by/ $4.0 /)$.
Abstract: 3D printing technologies offer significant advantages over conventional manufacturing technologies for objects with complicated shapes. This technology provides the potential to easily manufacture barrier windows with a low reflection in a wide frequency band. Several 3D printing methods were examined for this purpose, and the dielectric properties of the various types of materials used for 3D printing were experimentally studied in the frequency range $26-190 \mathrm{GHz}$. These measurements show that the styrene-butadiene-styrene and polyamide plastics are suitable for broadband low-reflection windows for low-to-medium-power microwave applications. Two barrier windows with optimized surface shapes were printed and tested. Results demonstrate that the studied technique can fabricate windows with a reflection level below $-18 \mathrm{~dB}$ in the frequency band up to $160 \mathrm{GHz}$. Studied windows can be used for spectroscopic tasks and other wideband microwave applications.

Keywords: dielectric properties; low-reflection barrier windows; broadband window; microwaves; terahertz radiation

\section{Introduction}

Additive manufacturing technologies have great potential for industry, science, and technology. There are a number of tasks that are difficult or almost impossible to implement with traditional fabrication methods. In particular, 3D printing from dielectric materials is a highly convenient and cheap tool for prototyping and manufacturing radiofrequency components. It creates a method of readily obtaining components with a sophisticated surface shape. For low-reflection microwave windows, a subwavelength grating with a specially designed shape at both sides of the window disk could significantly reduce the reflection coefficient of incident radiation in a wide frequency band [1]. This paper explores the possibility of using 3D printing to make millimeter-wave barrier windows that can operate in a wide frequency range.

Currently, there are several areas in which windows with the broadband transmission of low-power microwave radiation are used. These areas include molecular gas spectroscopy and DNP/NMR spectroscopy [2,3], measurements of fine positronium structure [4], which require both broadband-tunable microwave radiation sources such as backward-wave oscillators, gyrotrons, orotrons, and input windows in the working chamber of the spectrometer. Other examples are the radiometers and geophysical instruments used for atmospheric transparency studies, which require windows with a low reflection coefficient at different frequencies, corresponding to atmospheric transparency windows, with a bandwidth up to $20 \mathrm{GHz}$ [5]. The cryogenic resonator complex requires an even wider frequency band [6], which needs a low reflection from the input and output windows in the entire operating frequency band (50-500 GHz). Reflections from windows lead to spurious interference, which ultimately reduces the sensitivity of the spectrometer. Such 
devices are currently equipped with lenses with concentric triangle-shaped grooves, which are noticeably worse in terms of reflections compared to the alternative surfaces considered in this paper [7]. At the same time, it is well known that reflections dramatically affect the gyrotron operation regime [8]. For all the mentioned applications, the radiation power does not exceed a few watts, which allows one to use windows made of polymers without the risk of overheating.

There are different methods to obtain the broadband transmission of microwave radiation through the windows. The almost reflectionless transmission of a linearly polarized wave can be achieved for the Brewster-angle disk; however, this output suffers from an inefficient use of space, which may be especially important for divergent wave beams and some distortion of the transverse field structure [9]. Polymers can be mixed with nanoparticles to produce a multilayer dielectric coating [10]. Metamaterial devices and gradient index photonic structures are also used to reduce reflection [11]. Another known method considered in this paper is the use of windows with a surface grating of a specially optimized shape, providing a significant reduction in reflection [12]. For the manufacture of such a surface, it is highly convenient to use $3 \mathrm{D}$ printing. Besides low cost and time consumption, 3D printing has no restrictions on the curvature of the surface compared to Computerized Numerical Control (CNC) machining. Furthermore, it has advantages in creating thin elements from relatively soft and brittle materials.

There are several different $3 \mathrm{D}$ printing technologies, each of which has its characteristics and uses its own types of polymers. Fused deposition modeling (FDM) technology [13] is readily available, easy to operate, and allows the use of a wide range of materials, including polyethylene, which has excellent properties in transmitting high-frequency radiation. However, this type of 3D printing is characterized by a low accuracy (up to 100 microns) and sufficiently pronounced layering, limiting the frequency range of applications. In addition, the print resolution in the transverse plane is limited by the diameter of the nozzle and the quality of the alignment. Selective laser sintering (SLS) technology [14], due to a similar process of plastic melting, also has a wide selection of materials. However, compared to FDM, it has higher accuracy and better resolution; a layer thickness of several tens of microns, a resolution in the transverse plane approximately equal to the size of the pellets of the plastic used. Finally, Photopolymer 3D printing (stereolithography, SLA) provides excellent print quality but currently proposes a limited choice of printing materials. In this paper, the SLS method was used for printing the studied window samples.

Currently, many different materials for 3D printing are presented on the market, and their number is constantly growing. Unfortunately, manufacturers usually do not provide information on the dielectric properties of materials, especially in the microwave, millimeter, and terahertz regions. In order to design the microwave components, the dielectric properties are of great importance, so the characterization of the materials used for additive manufacturing is needed.

This paper is organized as follows. In Section 2, the results of measurements of the dielectric properties of the commercially available plastics are described. In Section 3, the measurements of the transmission and reflection coefficients for 3D-printed windows with several surface shape profiles are presented, and the results are compared with conventionally manufactured windows. Section 4 discusses the transmitted power restrictions for the printed windows due to thermal properties. Finally, the results of the study are discussed and concluded in Section 5.

\section{Characterization of Dielectric Properties of Plastics Materials for 3D Printing}

To study the loss tangent and dielectric constant of the plastics, two independent methods were used to increase the reliability of the results. In the first method, a rod made of the investigated plastic was inserted into a rectangular waveguide. The reflection and transmission coefficients were measured, and the dielectric properties were calculated from the experimentally obtained frequency dependences. Formulas for reflection and transmission of the dielectric waveguide plug can be found in [15]. The scheme of the mea- 
surement is shown in Figure 1a. The measurements were made in the entire Ka frequency band $(26-40 \mathrm{GHz})$, and the measured reflection and transmission curves were matched by analytic curves calculated using the constant dielectric permittivity approximation. It can be seen in Figure $1 \mathrm{~b}$ that the constant dielectric permittivity approximation fits the measured data well.

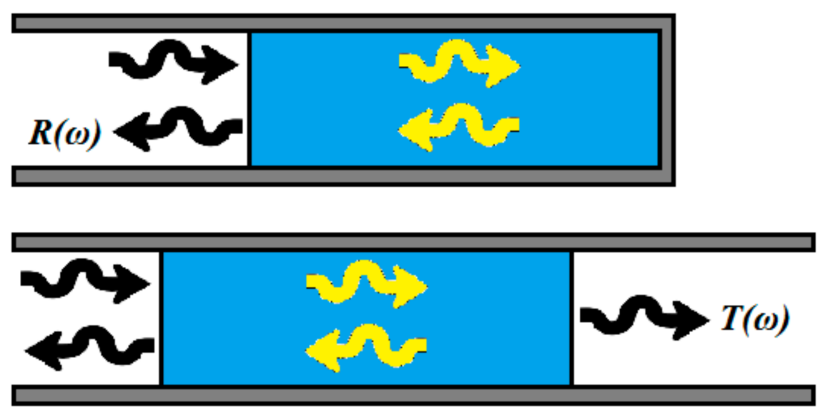

(a)
SBS 2

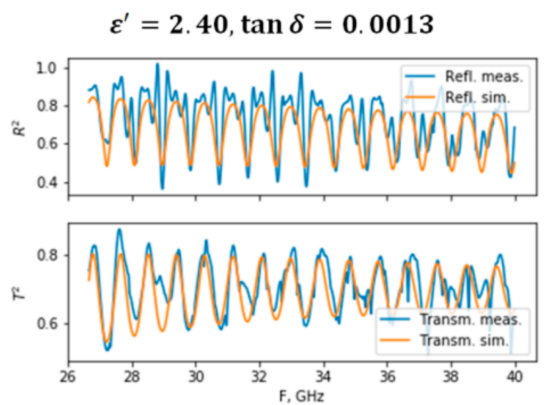

(b)

Figure 1. (a) The scheme of measurement of the reflection and transmission coefficients with the test sample in the form of the waveguide insert $7.2 \mathrm{~mm} \times 3.4 \mathrm{~mm} \times 100 \mathrm{~mm}(\mathbf{a}) ;(\mathbf{b})$ comparison of the measurement results and simulation data for SBS plastic in the frequency range $26-40 \mathrm{GHz}$.

During the experiments, polymer samples printed using various technologies were studied. Results of dielectric permittivity and loss tangent measurements of various plastics printed using different 3D printing technologies at $100 \%$ infill are presented in Table 1. The dispersion of dielectric permittivity is relatively small within the Ka-band. Analysis of experimental data shows that styrene-butadiene-styrene (SBS) and polyethylene terephthalate glycol (PETG) are the most suitable materials for windows due to low losses and moderate dielectric permittivity. Different samples of PETG and SBS were printed using filaments from different manufacturers and showed minor differences. However, we note that these types of plastics were only suitable for FDM technology printers, which provided fair accuracy and thus were not applicable at sub-terahertz frequencies but sufficient for frequencies of several tens of GHz. This method and this plastic were used earlier, in particular, to print a two-dimensional Bragg resonator operating in the frequency range 55-65 GHz, and the measurement results were in good agreement with the theory [16]. Since the windows for spectroscopic and atmospheric measurements are also required at higher frequencies, the study of the applicability of FDM printing for these purposes is of interest.

Table 1. Dielectric properties of the 3D-printed samples in Ka-band.

\begin{tabular}{cllc}
\hline Plastic & Printer & Re $\varepsilon$ & $\tan \delta$ \\
\hline $\begin{array}{c}\text { Polyethylene terephthalate } \\
\text { glycol (PETG) 1 }\end{array}$ & FDM & 2.31 & $1.5 \times 10^{-3}$ \\
PETG 2 & FDM & 2.47 & $1.6 \times 10^{-3}$ \\
Polylactic Acid (PLA) & FDM & 2.27 & $6.2 \times 10^{-3}$ \\
Sterol-butadiene-sterol (SBS) & FDM & 2.22 & $1.6 \times 10^{-3}$ \\
1 & FDM & 2.40 & $1.3 \times 10^{-3}$ \\
SBS 2 & SLA & 1.8 & $3.3 \times 10^{-2}$ \\
Visijet SL Clear & SLA & 1.8 & $3.5 \times 10^{-2}$ \\
Visijet SL Flex & SLA & 1.8 & $3.5 \times 10^{-2}$ \\
Visijet SL Hi-Temp & SLS & 1.7 & $3.5 \times 10^{-3}$ \\
Polyamide & & &
\end{tabular}

The second method for measuring the properties of dielectrics was to place samples in the form of flat disks between the mirrors of a high-quality open two-mirror Fabry-Perot 
resonator. By measuring the quality factor of an empty cavity and a cavity with a dielectric insert, it is possible to measure the properties of the test sample with high accuracy [17]. These measurements were made for selected materials and frequencies up to $185 \mathrm{GHz}$. The real part of the dielectric permittivity is the same as in the Ka-band measurements. The scheme of the measurement setup is shown in Figure 2a. The loss tangent data for plastic used for FDM printing (SBS) and plastic used for SLS printing (polyamide) is shown in Figure 2b.

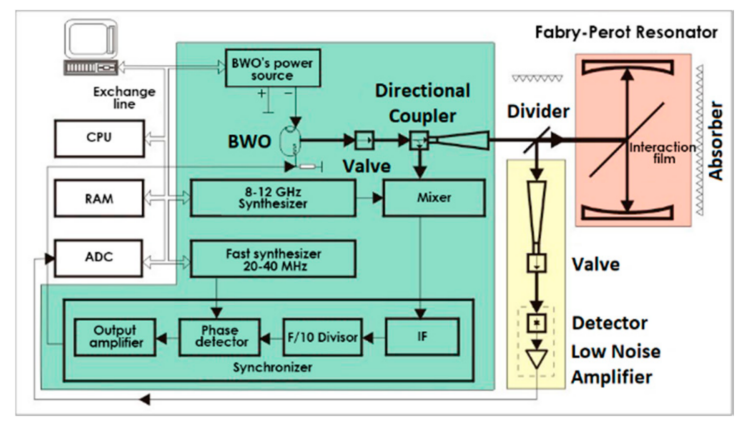

(a)

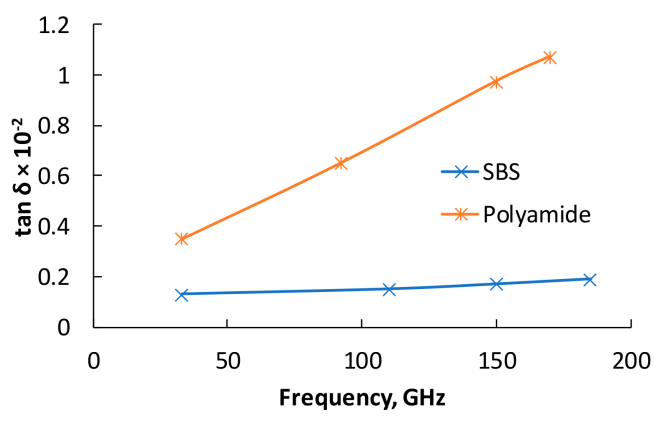

(b)

Figure 2. (a) The scheme of measurement of dielectric properties with the test sample in the form of the disk in the quasioptical resonator; (b) loss tangent of the SBS and polyamide.

The polyamide plastic for SLS printers has a somewhat higher absorption than SBS and PETG. Therefore, it could be an optimal solution for some applications as a trade-off between the higher losses and better printing quality of the SLS method.

The photopolymer materials have significantly larger losses than polyamide (tangent delta higher than 0.03). However, we consider these materials a good solution for highfrequency applications with a 1 milliwatt or lower level of microwave power, since SLA printing allows much better print accuracy and surface quality.

\section{Reflection Measurements for 3D-Printed Prototype Barrier Windows}

For the experimental study of the broadband windows prototypes, we chose SLS printing from polyamide due to low losses and high manufacturing accuracy, which allow the creation of small-scale structures suitable for devices operating at frequencies of several hundred GHz. The sizes of the subwavelength antireflection structures were chosen to produce fine details by the selected printing method adequately. These structures should perform well when half of the wavelength is bigger than the period of the structure because there could be no \pm 1 st order diffraction scattering in these conditions. However, the performance at higher frequencies might degrade faster or slower depending on the shape of the elements. This paper considers the two variants of known antireflection subwavelength gratings at window disks for additive manufacturing. The first variant of the surface shape is a periodic array of pyramids with a base size smaller than the wavelength [18]. The shape of the pyramidal grating is shown in Figure 3a. The advantages of such a surface are a weak dependence of the reflection coefficient on the frequency and polarization of the incident radiation. The base side of the pyramids was chosen to be $1 \mathrm{~mm}$, and the height was $2 \mathrm{~mm}$. Simulations show that this corrugation applied to both surfaces of the polyamide window disk and provided reflections of less than $-20 \mathrm{~dB}$ in the frequency band wider than one octave. For comparison, the flat polyamide disk had reflections of up to $-9 \mathrm{~dB}$ in this band. The second option is a one-dimensional periodic corrugation of a special shape, proposed in [19]. This profile is optimized to minimize the reflection of the polarization with the direction of the electric field orthogonal to the groove direction at the frequency range of $60-160 \mathrm{GHz}$. The shape of the grooves is shown in Figure $3 b$. The period and depth of one-dimensional corrugation are $2 \mathrm{~mm}$ and $2.5 \mathrm{~mm}$, 
respectively. The corrugation profile was optimized for one linear polarization only, and the reflection coefficient was less than $-20 \mathrm{~dB}$ for frequencies below $105 \mathrm{GHz}$.

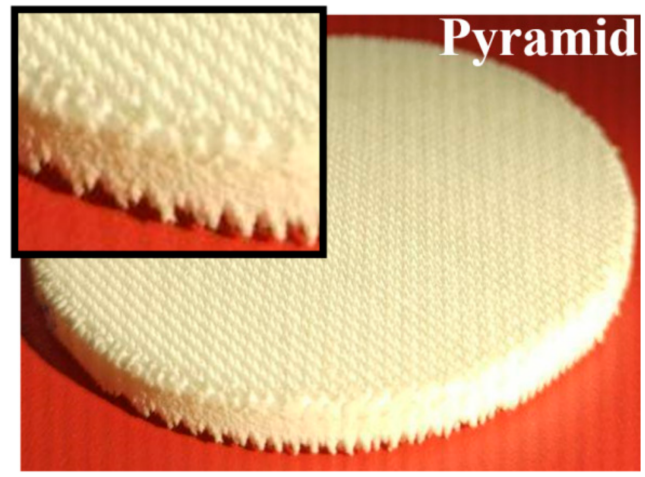

(a)

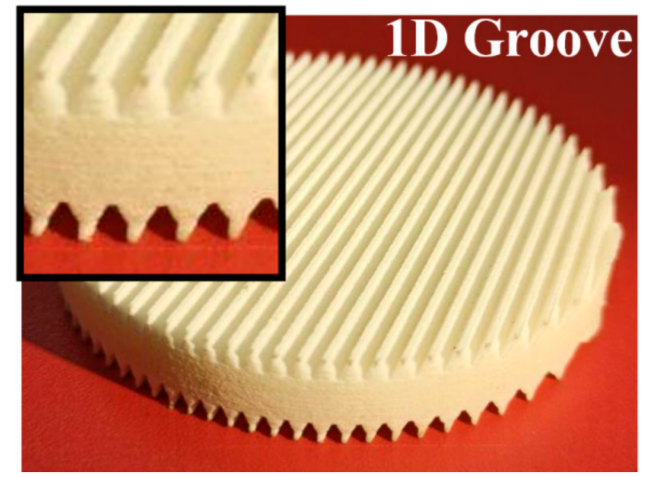

(b)

Figure 3. 3D-printed windows with (a) pyramids; (b) one-dimensional grating of special shape.

Three discs were printed: the first one had a flat surface on both sides, the other two had gratings of the tested shape on both sides. The reflection coefficients were obtained by two measurement setups by a vector network analyzer with a step of $30 \mathrm{MHz}$ in the bands of 75-110 GHz and 130-160 GHz, where the disks with optimized surface shapes calculated reflection minima. The window disks were attached to the output of the corrugated tapers providing the gaussian wave beam flat phase front (Figure 4a). The 0 - $\mathrm{dB}$ level of the setup was set using the flat mirror closing the end of the taper, and the minimum sensitivity limit was set as the reflection from the open end of the taper.

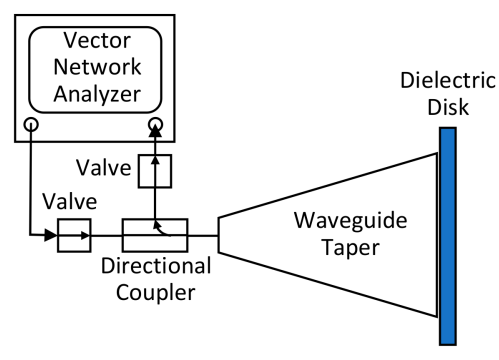

(a)

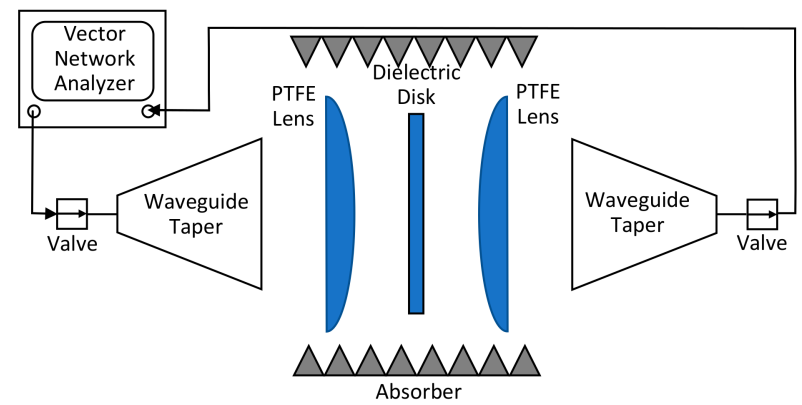

(b)

Figure 4. Schematics of the setups used to measure the dielectric disks parameters: (a) reflection measurements; (b) transmission measurements.

Reflection measurement results are shown in Figures 5-7 for the flat-surface disk, disk with pyramids, and disk with one-dimensional corrugation, correspondingly. The disk with a one-dimensional corrugation of the surface was measured for both orthogonal linear polarizations. However, the results for the second polarization are significantly worse than for the optimal polarization. The measured reflection coefficient is below $-18 \mathrm{~dB}$ for both corrugated disks in the frequency ranges of 75-110 GHz and 130-160 GHz. In contrast, the flat disk has a much narrower band with low reflections $(10 \mathrm{GHz}$ at the level of $-20 \mathrm{~dB}$ ). Note that the option with pyramids is more advantageous if radiation of arbitrary polarization is required, while the option with one-dimensional corrugation is better for specific linear polarization. The one-dimensional corrugation works better for lower frequencies but (due to its larger period) is worse for higher frequencies. Due to the high sensitivity of this shape to manufacturing tolerances, the measured reflection of 
one-dimensional corrugation significantly deviates from the calculated one, which is also noted in [19].

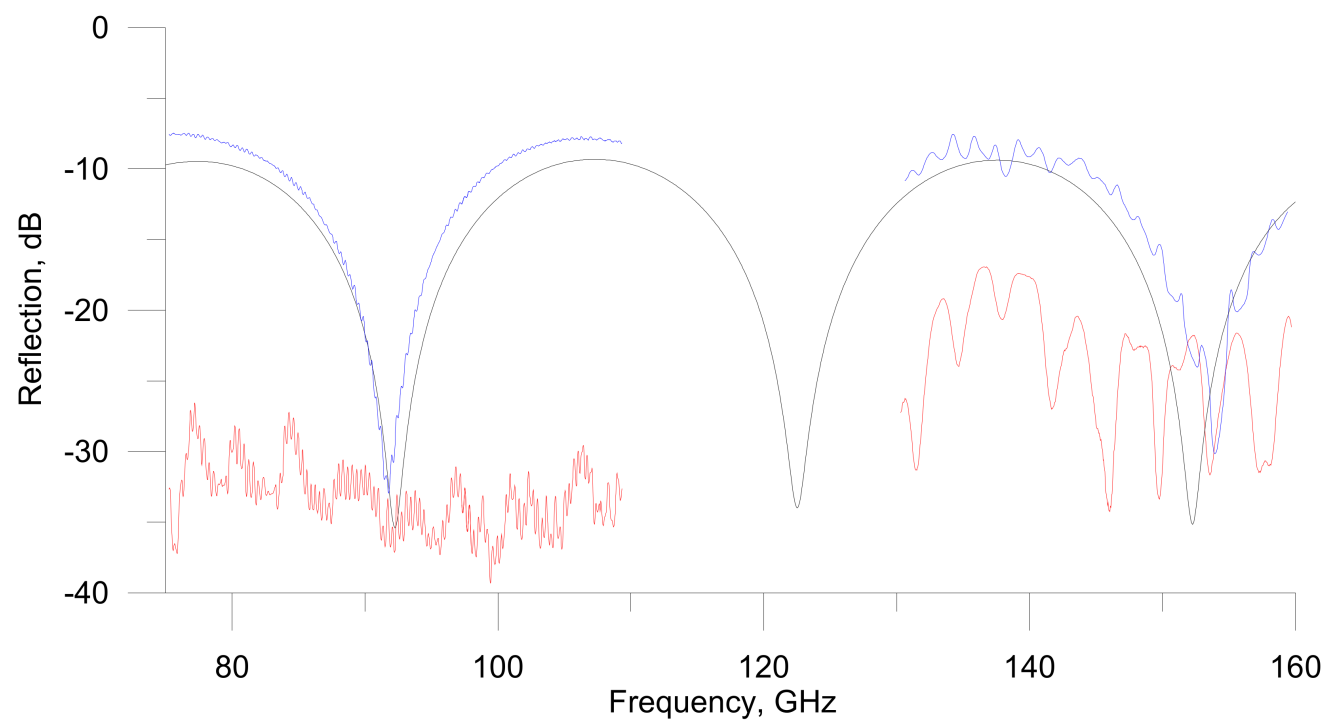

Figure 5. Reflection from the 3D-printed disk with a flat surface on both sides. The black line corresponds to the numerical simulation in CST Studio. The blue line is the measured reflection coefficient, and the red line is the lower sensitivity limit of the measurement setup.

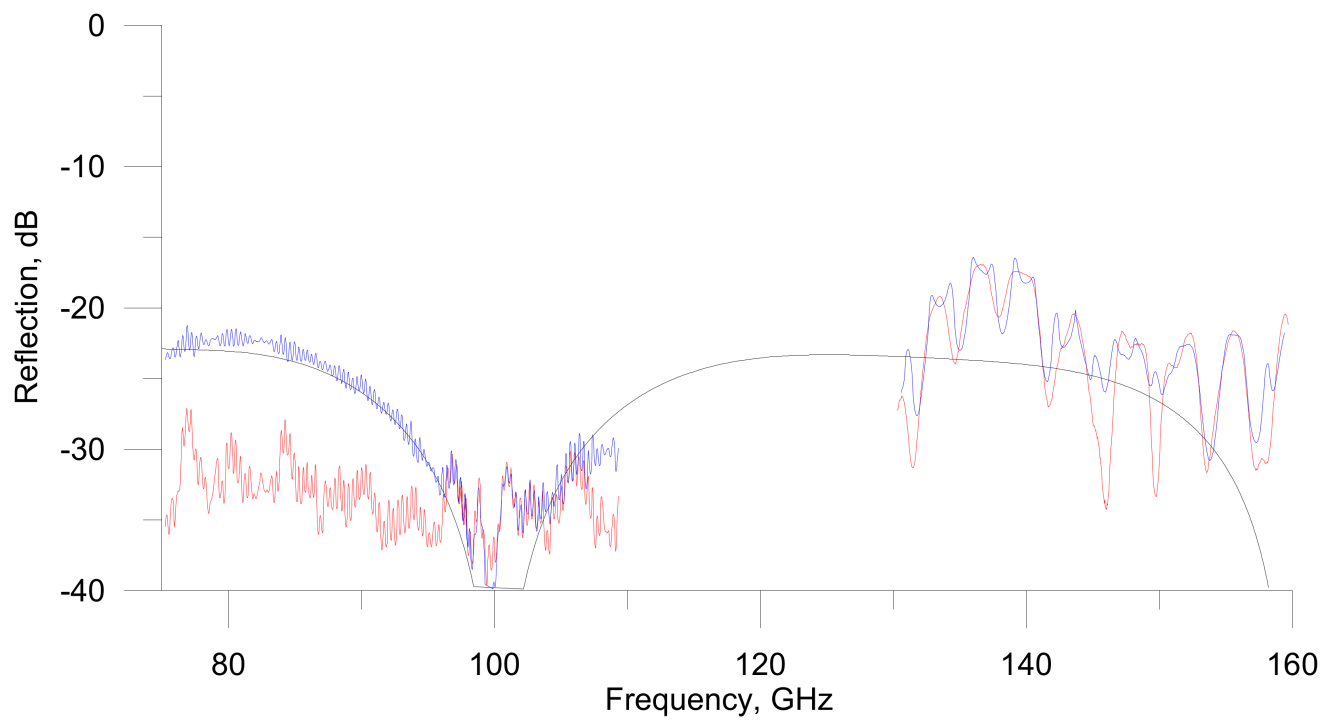

Figure 6. Reflection from the 3D-printed disk with pyramids on both sides. The black line corresponds to the numerical simulation in CST Studio. The blue line is the measured reflection coefficient, and the red line is the lower sensitivity limit of the measurement setup.

To measure wideband transmission, we used a quasioptical setup consisting of a vector network analyzer (VNA), a pair of tapers with PTFE lenses on adaptors, and an air gap between them (Figure 4b). Disks were placed in the center of the air gap, which is also the position of the Gaussian beam waist. The transmission through the two thinner disks is presented in Figure 8. The flat disk has a thickness of $3.15 \mathrm{~mm}$, and the average thickness of the disk with pyramids is $3.33 \mathrm{~mm}$. The low-reflection disk has a significantly better transmission and is very close to the maximum transmission predicted using the measured loss tangent of the polyamide. The disk with one-dimensional corrugation is not shown because it has high dielectric losses in this frequency band due to its bigger average thickness of $7.5 \mathrm{~mm}$. The oscillations of the flat-sided disk transmission are caused 
by Fabry-Perot resonances inside the disk (period approximately $30 \mathrm{GHz}$ ) and resonances between the disk and one of the lenses (approximately $4 \mathrm{GHz}$ ). The reflection from the tapers causes fast oscillations (approximately $1 \mathrm{GHz}$ ).

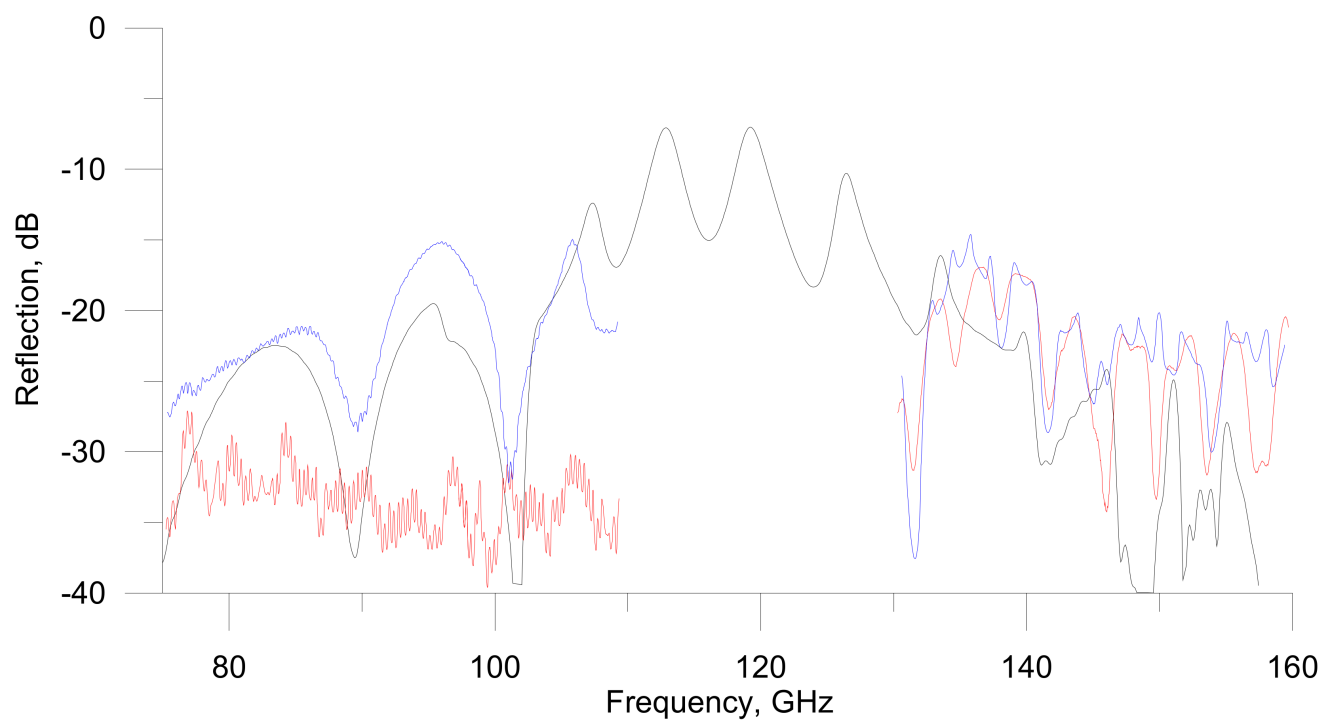

Figure 7. Reflection from the 3D-printed disk with special one-dimensional corrugation on both sides. The black line corresponds to the numerical simulation in CST Studio. The blue line is the measured reflection coefficient, and the red line is the lower sensitivity limit of the measurement setup.

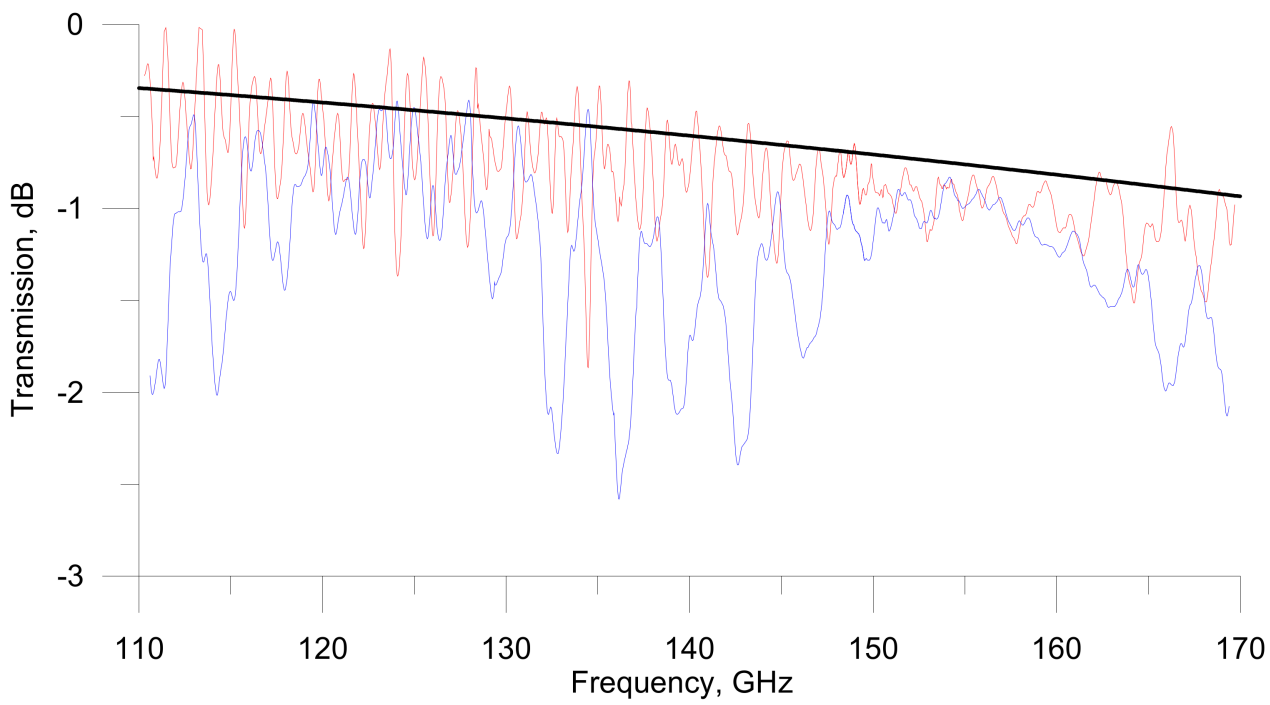

Figure 8. Transmission through the disks. The red line corresponds to the disk with pyramids on both sides, and the blue line corresponds to the flat-surface disk. The black line shows dielectric losses calculated in the $3.33 \mathrm{~mm}$ thick polyamide disk with zero reflections.

It is interesting and instructive to compare the results obtained for 3D-printed windows with the earlier results for windows with the same shape profiles but manufactured by traditional mechanical methods. Thus, the disk made from raflon (radiation-modified PTFE) by CNC machining had reflections lower than $-20 \mathrm{~dB}$ in the frequency range of $50-200 \mathrm{GHz}$ [19], which is very similar to the results presented in this paper. Therefore, we conclude that the current additive technologies are competitive with traditional manufacturing methods for dielectric microwave components at the $1 \mathrm{~mm}$ and longer waves. 


\section{Estimation of the Applicability of the Considered Materials}

Based on the obtained values of the real and imaginary parts of the dielectric permittivity, as well as the mechanical and thermal parameters of the tested materials given in [20], it is possible to determine the losses and temperature conditions of a window that can withstand atmospheric pressure, depending on its diameter and the supplied microwave power. When considering the heat problem, the transverse dependence of the microwave radiation intensity on the radius was taken as a Gaussian wave beam with a width of 0.64 of the window radius. Numerical modeling was performed with the following parameters to represent many plastics with similar properties: $n=1.5$, emissivity $\varepsilon=0.9$, and the thermal conductivity of plastic $\mathrm{k}=0.4 \mathrm{~W} \cdot \mathrm{m}^{-1} \mathrm{~K}^{-1}$. The dielectric loss tangent was taken as slightly larger than the best-tested plastic $\delta=0.002$. The temperature of the cooled edge of the window (ambient temperature) was $\mathrm{T}_{0}=20^{\circ} \mathrm{C}$, with a convection coefficient of $\mathrm{h}$ $=10 \mathrm{~W} \cdot \mathrm{m}^{-2} \cdot \mathrm{K}^{-1}$. Several window diameters were considered between $1 \mathrm{~cm}$ and $10 \mathrm{~cm}$ with different thicknesses from $1 \mathrm{~mm}$ to $10 \mathrm{~mm}$, and the wavelength was set as $\lambda_{0}=3 \mathrm{~mm}$, which corresponded to the $100 \mathrm{GHz}$ base frequency. Dielectric losses are proportional to the frequency; therefore, the maximum power for any other frequency can be calculated by multiplying the ratio of the base frequency to the target frequency. Numerical modeling of the dielectric disk heating by a Gaussian beam was performed in COMSOL Multiphysics. The reflections of the beam on disk surfaces were neglected, assuming the antireflection surfaces. We simulated maximum beam power, which can be transmitted through the disk, given that the maximum stationary temperature is $120^{\circ} \mathrm{C}$. The decimal logarithm of the maximum transmitted continuous wave (CW) power value in Watts is presented in Figure 9 , e.g., a disk with diameter $100 \mathrm{~mm}$ and thickness $10 \mathrm{~mm}$ can withstand approximately $150 \mathrm{~W}$ of $\mathrm{CW}$ transmitted power at a frequency of $100 \mathrm{GHz}$ without convective cooling.

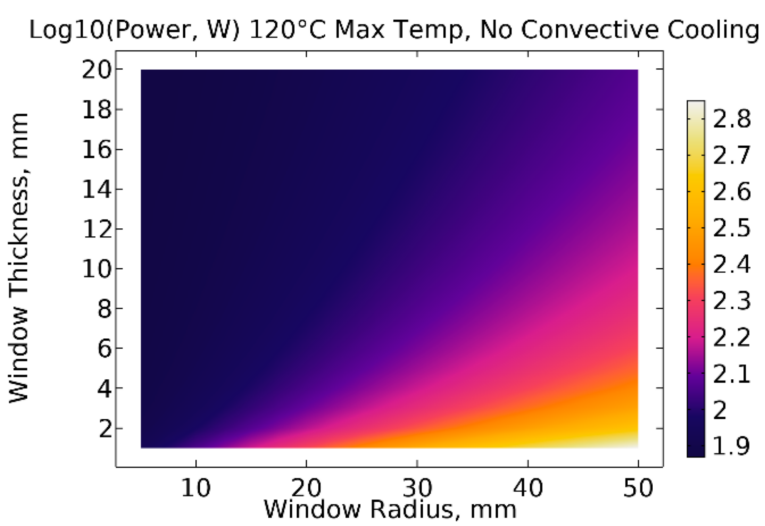

(a)

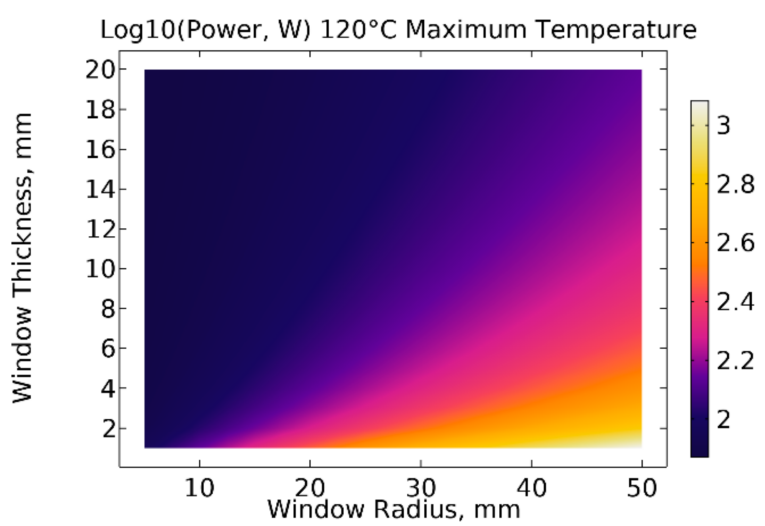

(b)

Figure 9. Simulation results for maximum $100 \mathrm{GHz} \mathrm{CW}$ transmitted power through the polymer windows without overheating. (a) the decimal logarithm of the maximum power value in watts without convective cooling (vacuum at both sides); (b) decimal logarithm of the maximum power value in watts with convective air cooling on one side.

\section{Conclusions}

Current 3D printing technologies allow easy-to-manufacture dielectric microwave components, such as broadband barrier windows with low reflections. In this paper, the dielectric properties of the materials used in various $3 \mathrm{D}$ printing methods were measured. The most suitable materials were found to be useful in microwave systems with a frequency of up to several hundred gigahertz and a power of up to several tens of watts. The analytical calculations and numerical simulations verify the use of the studied materials for microwave devices with a power of about 100-200 watts.

Barrier windows with surface shapes specially optimized for low reflection in a wide frequency band were printed and examined at low power. The printed windows provided a reflection coefficient below $-18 \mathrm{~dB}$ at frequencies of up to $160 \mathrm{GHz}$, making it possible 
to use in microwave devices that require the reception/transmission of a signal in a wide frequency range. We conclude that the current additive technologies are competitive with traditional manufacturing methods for dielectric microwave components at $1 \mathrm{~mm}$ and for longer waves.

Author Contributions: Conceptualization, M.Y.G., M.D.P. and D.I.S.; methodology, M.D.P. and D.I.S.; investigation, M.D.P., D.I.S., V.V.P., V.I.B. and S.V.M.; writing-original draft preparation, M.D.P.; writing - review and editing, D.I.S.; project administration, M.Y.G. All authors have read and agreed to the published version of the manuscript.

Funding: This research was funded by Russian Science Foundation, grant number 21-19-00877.

Data Availability Statement: Data available on request from the authors.

Conflicts of Interest: The authors declare no conflict of interest.

\section{References}

1. Mirotznik, M.S.; Good, B.L.; Ransom, P.; Wikner, D.; Mait, J.N. Broadband antireflective properties of inverse motheye surfaces. IEEE Trans. Antennas Propag. 2010, 58, 2969-2980. [CrossRef]

2. Idehara, T.; Mitsudo, S.; Sabchevski, S.; Glyavin, M.Y.; Ogawa, I. Gyrotron FU series—Current status of development and applications. Vacuum 2001, 62, 123-132. [CrossRef]

3. Idehara, T.; Sabchevski, S.P.; Glyavin, M.; Mitsudo, S. The gyrotrons as promising radiation sources for THz sensing and imaging. Appl. Sci. 2020, 10, 980. [CrossRef]

4. Fedotov, A.E.; Rozental, R.M.; Zotova, I.V.; Ginzburg, N.S.; Sergeev, A.S.; Tarakanov, V.P.; Glyavin, M.Y.; Idehara, T. Frequency tunable sub-THz gyrotron for direct measurements of positronium hyperfine structure. J. Infrared Millim. Terahertz Waves 2018, 39, 975-983. [CrossRef]

5. Nosov, V.I.; Bolshakov, O.S.; Bubnov, G.M.; Vdovin, V.F.; Zinchenko, I.I.; Marukhno, A.S.; Nikiforov, P.L.; Fedoseev, L.I.; Shvetsov, A.A. A dual-wave atmosphere transparency radiometer of the millimeter-wave range. Instrum. Exp. Tech. 2016, 59, 374-380. [CrossRef]

6. $\quad$ Parshin, V.V.; Serov, E.A.; Bubnov, G.M.; Vdovin, V.F.; Koshelev, M.A.; Tretyakov, M.Y. Cryogenic resonator complex. Radiophys. Quantum Electron. 2014, 56, 554-560. [CrossRef]

7. Lamb, J.W. Cross-Polarisation and astigmatism in matching grooves. Int. J. Infrared Millim. Waves 1996, 17, 2159-2165. [CrossRef]

8. Glyavin, M.Y.; Zapevalov, V.E. Reflections influence on the gyrotron oscillation regimes. Int. J. Infrared Millim. Waves 1998, 19, 1499-1511. [CrossRef]

9. Li, Q.; Vernon, R.J. Reflection and transmission of a Gaussian beam from a dielectric window at the Brewster angle. In Proceedings of the 2005 Joint 30th International Conference on Infrared and Millimeter Waves and 13th International Conference on Terahertz Electronics, Williamsburg, VA, USA, 19-23 September 2005; Volume 2, pp. 513-514.

10. Cai, B.; Chen, H.; Xu, G.; Zhao, H.; Sugihara, O. Ultra-Broadband THz antireflective coating with polymer composites. Polymers 2017, 9, 574. [CrossRef] [PubMed]

11. Chen, Y.W.; Zhang, X.C. Anti-Reflection implementations for terahertz waves. Front. Optoelectron. 2014, 7, 243-262. [CrossRef]

12. Bräuer, R.; Bryngdahl, O. Design of antireflection gratings with approximate and rigorous methods. Appl. Opt. 1994, 33, 7875-7882. [CrossRef] [PubMed]

13. 3D Printing \& Additive Manufacturing. Available online: https://www.stratasys.com/ (accessed on 29 June 2021).

14. Beaman, J.J.; Deckard, C.R. Selective Laser Sintering with Assisted Powder Handling. U.S. Patent 4938816A, 3 July 1990.

15. Rinkevich, A.B.; Perov, D.V.; Ryabkov, Y.I. Transmission, reflection and dissipation of microwaves in magnetic composites with nanocrystalline finemet-type flakes. Materials 2021, 14, 3499. [CrossRef] [PubMed]

16. Ginzburg, N.S.; Peskov, N.Y.; Zaslavsky, V.Y.; Kocharovskaya, E.R.; Malkin, A.M.; Sergeev, A.S.; Baryshev, V.R.; Proyavin, M.D.; Sobolev, D.I. 2D Bragg resonators based on planar dielectric waveguides (from theory to model-based testing). Semiconductors 2019, 53, 1282-1286. [CrossRef]

17. Vlasov, S.N.; Koposova, E.V.; Mazur, A.B.; Parshin, V.V. On permittivity measurement by a resonance method. Radiophys. Quantum Electron. 1996, 39, 410-415. [CrossRef]

18. Ma, J.Y.L.; Robinson, L.C. Night moth eye window for the millimetre and sub-millimetre wave region. Opt. Acta Int. J. Opt. 1983, 30, 1685-1695. [CrossRef]

19. Vlasov, S.N.; Koposova, E.V.; Kornishin, S.Y. Wideband windows for millimeter- and submillimeter-wave vacuum devices. Radiophys. Quantum Electron. 2020, 63, 115-123. [CrossRef]

20. Laureto, J.; Tomasi, J.; King, J.A.; Pearce, J.M. Thermal properties of 3-D printed polylactic acid-metal composites. Prog. Addit. Manuf. 2017, 2, 57-71. [CrossRef] 